\title{
Is the giant elliptical galaxy NGC 5018 a post-merger remnant?
}

\author{
L. M. Buson ${ }^{1}$, F. Bertola ${ }^{2}$, A. Bressan ${ }^{1}$, D. Burstein ${ }^{3}$, and M. Cappellari ${ }^{4}$ \\ 1 INAF - Osservatorio Astronomico di Padova, vicolo dell'Osservatorio 5, 35122 Padova, Italy \\ e-mail: buson@pd.astro.it \\ 2 Dipartimento di Astronomia, Università di Padova, vicolo dell'Osservatorio 2, 35122 Padova, Italy \\ 3 Department of Physics \& Astronomy, Arizona State University, Tempe, AZ, 85287-1504, USA \\ ${ }^{4}$ Leiden Observatory, Postbus 9513, 2300 RA Leiden, The Netherlands
}

Received 16 February 2004 / Accepted 13 June 2004

\begin{abstract}
NGC 5018, one of the weakest UV emitters among giant ellipticals (gE) observed with IUE, appears to consist of an optical stellar population very similar to that of the compact, dwarf elliptical M32, which is several magnitudes fainter in luminosity than NGC 5018 and whose stellar population is known to be $\sim 3$ Gyr old. Here we show that the mid-UV spectra of these two galaxies are also very similar down to an angular scale hundreds times smaller than the IUE large aperture (as probed by HST/FOS UV spectra obtained through $0.86^{\prime \prime}$ apertures). This implies a reasonably close match of the populations dominating their mid-UV light (namely, their main-sequence turnoff stars). These data indicate that NGC 5018 has, in its inner regions, a rather uniform dominance of a $\sim 3$ Gyr-old stellar population, probably a bit different in metallicity from M 32 . Combined with the various structures that indicate that NGC 5018 is the result of a recent major merger, it appears that almost all of stars we see in its center regions were formed about $3 \mathrm{Gyr}$ ago, in that merger event. NGC 5018 is likely the older brother of NGC 7252, the canonical gE-in-formation merger. As such, NGC 5018 is perhaps the best galaxy which can tell us how a merger works, after the fireworks subside, to form a gE galaxy today. For this reason alone, the stellar populations in NGC 5018 at all radii are worth studying in detail.
\end{abstract}

Key words. ultraviolet: galaxies - galaxies: elliptical and lenticular, cD - galaxies: individual: NGC 5018 galaxies: individual: M 32

\section{Introduction}

NGC 5018 is the dominant giant elliptical of a small group (Gourgolhon et al. 1992), whose distance is kinematically estimated between $40.8 \mathrm{Mpc}$ (Faber et al. 1989) and $39.9 \mathrm{Mpc}$ (Hyperleda Database; Paturel et al. 2003). An independent, almost identical estimate ( $\sim 39.8 \mathrm{Mpc})$ comes also from the observed light curve of its recent SN Ia 2002dj (Hutchings \& Li 2002), having adopted a total reddening $E(B-V) \sim 0.2$ along its line of sight (G. Pignata, private communication).

NGC 5018 is quite peculiar in two main respects. First, Schweizer et al. (1990) classified NGC 5018 as one its prime candidates for a recent major merger, assigning it a $\Sigma$ parameter of 5.15. Second, although it is morphologically classified as a gE, its nuclear optical spectrum distinguishes itself by having the weakest measurement of the absorption line index $\mathrm{Mg}_{2}$ for its velocity dispersion among the over $400 \mathrm{gEs}$ surveyed by the 7 Samurai $\left(\mathrm{Mg}_{2}=0.218 \pm 0.007\right.$; Davies et al. 1987). Its unusual properties brought it to our attention in the early 1990's, and the IUE spectrum we obtained (Bertola et al. 1993) lacks the prominent UV-upturn shortward of $\lambda 2000 \AA$, that is typical of old, metal-rich spheroids (Burstein et al. 1988).
Given these data, it is not surprising that some observers have suggested the existence of a fine-tuned conspiracy, where young stars and heavy dust absorption conspire to dilute the underlying $\mathrm{Mg}_{2}$ line strength index, also turning a flat, young-star-dominated UV energy distribution into the observed NGC 5018 UV-weak spectrum (Carollo \& Danziger 1994). However, this scenario is not consistent with the finding that the average internal reddening is as low as $E(B-V) \sim 0.02$, within the $10^{\prime \prime} \times 20^{\prime \prime}$ IUE aperture (Buson et al. 2001). In the present Research Note we re-examine the stellar population of NGC 5018 by comparing its UV spectra (obtained with both HST/FOS and IUE) with similar spectra of M32. In so doing, we believe we can now identify NGC 5018 as the best candidate we have for the result of a major merger forming a gE galaxy in today's universe.

\section{The UV evidence}

Bertola et al. (1993) found an unexpectedly good match between the far-UV spectra of NGC $5018\left(M_{B}=-21.52 ; \sigma=\right.$ $212 \mathrm{~km} \mathrm{~s}^{-1}$; Prugniel \& Simien 1996) and of the cE dwarf 


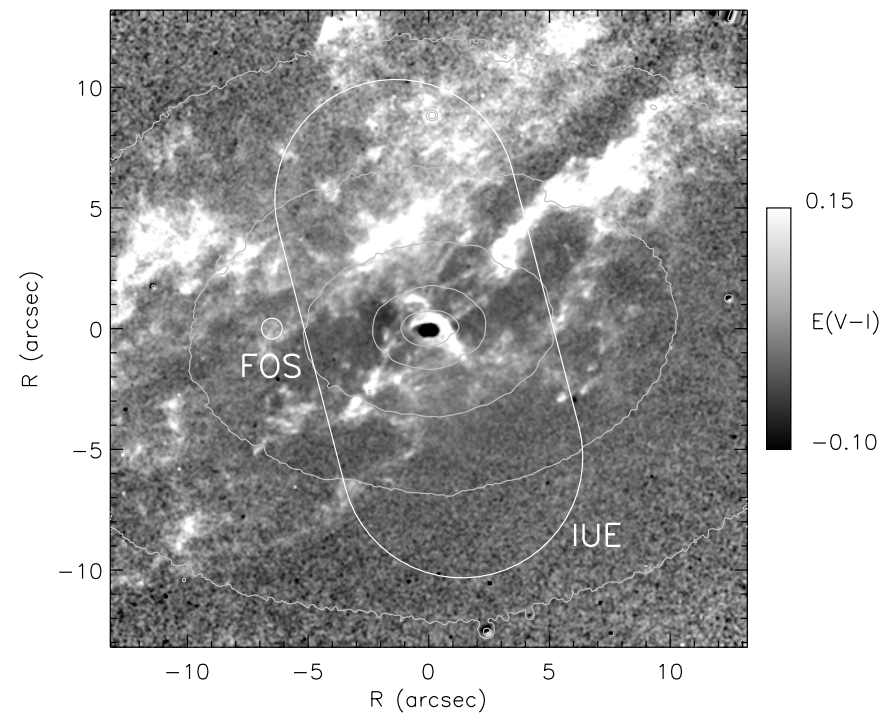

Fig. 1. Color excess map $E(V-I)$ of the center of NGC 5018, as obtained from HST/WFPC2 observations, after subtracting the underlying stellar color gradient of Fig. 2. Superimposed on this field are the locations of the IUE aperture (approximately $10^{\prime \prime} \times 20^{\prime \prime}$ in size), as well as of the $0.86^{\prime \prime}$ diameter circular aperture used for the HST/FOS off-nucleus observation. The gray contours levels represent the $I$-band galaxy surface brightness, in steps of $1 \mathrm{mag} \operatorname{arcsec}^{-2}$. North is up and east is to the left.

galaxy M32, four magnitudes less luminous $\left(M_{B}=-17.76\right.$; $\sigma=74 \mathrm{~km} \mathrm{~s}^{-1}$ ). Such a similarity includes the lack of farUV flux, comparable mid-UV spectral shape, as well as similar $\mathrm{Mg}_{2}$ line-strength index values in the optical $(0.196 \pm 0.007$; for M 32; Trager et al. 1998, compared to 0.218 for NGC 5018). The HST/FOS spectra we discuss below show that mid-UV homogeneity of the two galaxy populations holds even at subarcsec angular scales. Any viable interpretation one wants to give to the origin of the stellar population of NGC 5018 must account for the rather uniform stellar population that is seen throughout its central regions.

Leonardi \& Worthey (2000) proposed that NGC 5018 is an an old, metal-rich elliptical which has undergone a major accretion event at some recent stage of its evolution. In this interpretation, NGC 5018 appears dominated by a stellar population similar to that of M 32, about 3 Gyr-old from current estimates (Trager et al. 2000; Terlevich \& Forbes 2002), which masks the old stellar population in this galaxy.

Interest in the puzzling stellar population content of NGC 5018 pushed one of us (DB) to include it in a HST-based spectroscopic survey carried out through the (circular) Faint Object Spectrograph 0.86" aperture (Ponder et al. 1998). This dataset included four exposures with the UV grating $\mathrm{G} 270 \mathrm{H}$ $(4 \times 2400 \mathrm{~s})$ and a single exposure (1020 s) with the UV/optical grating $\mathrm{G} 400 \mathrm{H}$, giving continuous spectral coverage from 2300 to $4800 \AA$. Luckily, the HST archives also contain an analogous FOS G270H+G400H set of spectra taken with the $0.86^{\prime \prime}$ square FOS aperture of the center of M32 (programme ID = 6636; PI: M. Gregg). It also consists of four $\mathrm{G} 270 \mathrm{H}$ exposures $(4 \times$ $1000 \mathrm{~s}$ ) and an additional, short-exposure (375 s) G400H spectrum. The net result is that we fortuituously have rare tools to

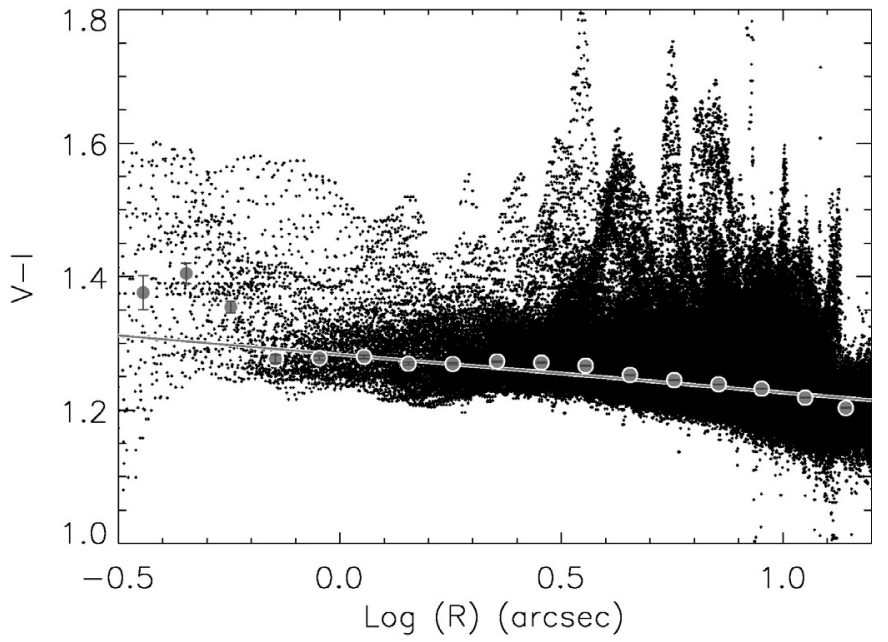

Fig. 2. Determination of the dust-corrected central $V-I$ color gradient from the HST/WFPC2 images, using the method of Cappellari et al. (2002). The black dots represent the color of the individual pixels in the PC1 CCD, as a function of their elliptical radius. The straight line is a robust fit which minimizes the sum of the absolute deviations of the points from the line, being thus almost insensitive to dust effects. The best fit, calibrated to the Johnson system following Dolphin $(2000)$ gives $(V-I)=(1.283 \pm 0.004)-(0.057 \pm 0.006) \times \log \left(\mathrm{r} / 1^{\prime \prime}\right)$, its zeropoint being not corrected for galactic reddening. Filled circles represent the median color computed in logarithmically spaced radial bins (formal errors are smaller than the symbol size).

probe directly the stellar content of these two galaxies over a wide range of angular scales.

In the case of NGC 5018 the FOS aperture was located off-nucleus, the result of an initially incorrect telescope position. As such, this UV spectrum corresponds to a region of the galaxy body outside the oval aperture of the previous IUE spectrum (though within the galaxy's effective radius $r_{\mathrm{e}} \sim 15^{\prime \prime}$; Alonso et al. 2003). Its final position, including also the fine adjustment due to the ACQ/PEAK pointing procedure, is $\sim 6^{\prime \prime}$ east of the nucleus, as shown in Fig. 1. The latter image represents the $E(V-I)$ color excess map of the center of NGC 5018, obtained by calibrating properly aligned, deep HST/WFPC2 archive observations of F. Schweizer (four, 1100-s F555W plus four, 1300-s F814W exposures), once the underlying stellar $(V-I)$ color gradient shown in Fig. 2 has been subtracted. The total eastward FOS aperture shift has been independently cross-checked by comparing the FOS spectrum level with the flux recorded at the same position in a UV WFPC2/F336W frame taken from a previous HST programme of ours (ID 5732).

In addition to these HST/FOS observations, IUE also observed both NGC 5018 and M32. The spectra discussed here consist of single, low-resolution LWP exposures obtained through the large, oval aperture centered on their nuclei and oriented at $\mathrm{PA}=14^{\circ}$ and $\mathrm{PA}=153^{\circ}$, respectively. The corresponding exposure times are 335 and $180 \mathrm{~min}$. 


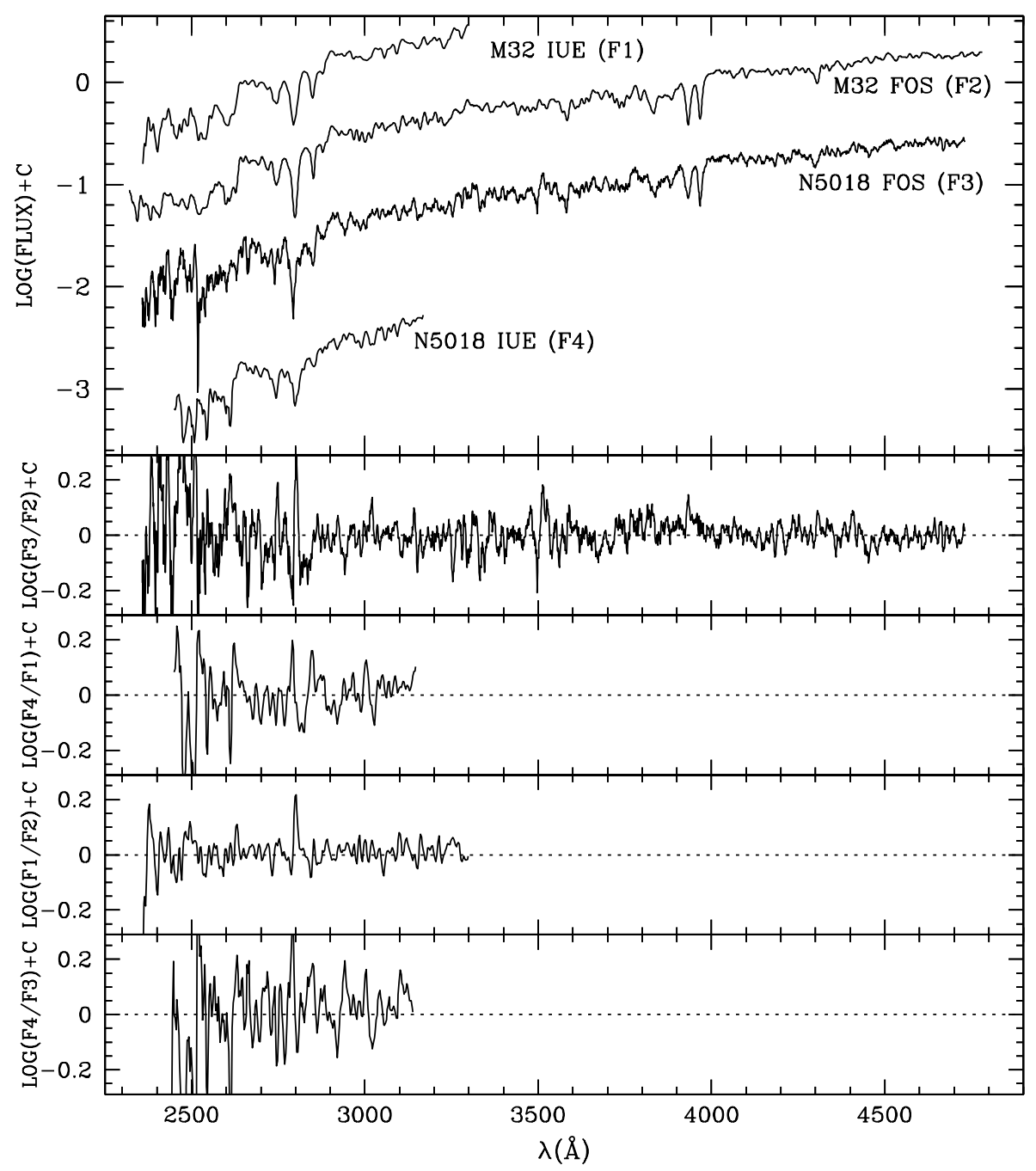

Fig. 3. Top panel: IUE spectra and FOS G270H+G400H spectra for the center region of M 32 and the bulge of NGC 5018. Lower panels: the divisions of each spectrum by each other, namely M32/FOS by NGC 5018/FOS, M 32/IUE by NGC 5018/IUE, M 32 FOS/M 32 IUE and NGC 5018 FOS/NGC 5018 IUE. All plots are given in either $\log F_{\lambda}+C$ or $\log \left[F_{\lambda}(X) / F_{\lambda}(Y)\right]+C$ vs. $\lambda$.

\section{Results}

The major outcome of these comparisons between the two galaxies is that the match (Fig. 3) of both the overall SED and the mid-UV triplet, i.e. of the absorption lines formed by the Mg II $2800 \AA$, Mg I $2852 \AA$ and the Fe I + II + Cr I feature at $2750 \AA$, well-known for showing rapid changes in relative strengths from late $B$ to late $F$ stars, holds over a wide range of angular sizes, from 10's of arc-seconds down to a $<1$ " field of view. As far as FOS spectra are concerned, a more quantitative appreciation of the match of NGC 5018 and M 32 SEDs shown in Fig. 3 can be obtained by the standard mid-UV indices listed in Table 1, measured after a proper velocity dispersion match of the original spectra. (Note: the apparent inversion of the $\mathrm{Ca}$ II $\mathrm{H}$ and $\mathrm{K}$ lines in the FOS spectrum of NGC 5018 is most likely due to an unfortunate cosmic ray, as this inversion does not correspond to the stellar population seen in the other parts of this spectrum.)

While the overall UV spectral energy distributions (SEDs) of M32 and NGC 5018 are very similar over a wide range of size scales, it is also clear from Table 1 and from the
Table 1. FOS ultraviolet spectral indices.

\begin{tabular}{lcccc}
\hline \hline \multicolumn{1}{c}{ Feature } & M 32 & Error & N5018 & Error \\
\hline 2600-3000 & 1.459 & 0.003 & 1.340 & 0.036 \\
MgWide & 0.285 & 0.002 & 0.400 & 0.025 \\
FeI+II+CrI & 0.351 & 0.010 & 0.275 & 0.108 \\
2609/2660 & 0.883 & 0.015 & 0.384 & 0.165 \\
MgII 2800 & 0.795 & 0.014 & 0.625 & 0.157 \\
MgI 2852 & 0.349 & 0.008 & 0.332 & 0.087 \\
2828/2921 & 0.510 & 0.005 & 0.690 & 0.058 \\
FeI 3000 & 0.199 & 0.003 & 0.117 & 0.028 \\
\hline
\end{tabular}

Adopted redshift $(1+z)=1.0093$ (NGC 5018); 0.9995 (M 32).

analysis of Leonardi \& Worthey (2000) that the stellar population of NGC 5018 is likely of somewhat different metallicity than that of M 32. However, as 3 Gyr-old stellar populations are dominated by the F-stars that define their main sequences, 
metallicity is a side issue in the match of the SEDs; it is the 3 Gyr-old ages of these two galaxies that dominate their SEDs.

\section{Conclusions}

This work shows that the stellar populations of the gE galaxy NGC 5018 and the dE galaxy M32 are of similar age ( $\sim 3$ Gyr-old), and this relatively young stellar population is spread rather uniformly within the central few kpc of NGC 5018. This evidence, coupled with the rather detailed evidence that indicates that NGC 5018 underwent a recent, major merger, strongly suggests that the young stellar population we see in this galaxy was the result of that major merger. As such, it is likely that NGC 5018 is the older brother of NGC 7252, which Schweizer (e.g., Schweizer 1982) has shown is at the tail-end of a major merger that is transforming its components into a gE galaxy now. As such, our observations raise a number of questions that bear on how such a major merger forms a gE galaxy a few billion years after the event. Chief among these questions is the issue of any evidence of an older stellar population in NGC 5018, or were literally most of the stars we see formed in the merger event? NGC 5018 is a galaxy whose stellar populations at all radii are worth a detailed study to gain a better understand how gE galaxies are formed via mergers today.

Acknowledgements. M.C. acknowledges support from a VENI grant awarded by the Netherlands Organization of Scientific Research (NWO).

\section{References}

Alonso, M. V., Bernardi, M., da Costa, L. N., et al. 2003, AJ, 125, 2307

Bertola, F., Burstein, D., \& Buson, L. M. 1993, ApJ, 403, 573

Burstein, D., Bertola, F., Buson, L. M., Faber, S. M., \& Lauer, T. R. 1988, ApJ, 328, 440

Buson, L. M., Cappellari, M., Bertola, F., \& Burstein, D. 2001, in Galaxy Disks and Disk Galaxies, ed. J. G. Funes S.J., \& E. M. Corsini, ASP Conf. Ser., 230, 435

Cappellari, M., Verolme, E. K., van der Marel, R. P., et al. 2002, ApJ, 578,787

Carollo, C. M., \& Danziger, I. J. 1994, MNRAS, 270, 743

Davies, R. L., Burstein, D., Dressler, A., et al. 1987, ApJS, 64, 581

Dolphin, A. E. 2000, PASP, 112, 1383

Faber, S. M., Wegner, G., Burstein, D., et al. 1989, ApJS, 69, 763

Gourgoulhon, E., Chamaraux, P., \& Fouque, P. 1992, A\&A, 255, 69

Hutchings, D., \& Li, W. D. 2002, IAU Circ., 7918, 1

Leonardi, A. J., \& Worthey, G. 2000, ApJ, 534, 650

Paturel, G., Petit, C., Prugniel, Ph., et al. 2003, A\&A, 412, 45

Ponder, J. M., Burstein, D., O'Connell, R. W., et al. 1998, ApJ, 116, 2297

Prugniel, P., \& Simien, F. 1996, A\&A, 309, 749

Schweizer, F. 1982, ApJ, 252, 455

Schweizer, F., Seitzer, P., Faber, S. M., et al. 1990, ApJ, 364, L33

Terlevich, A. I., \& Forbes, D. A. 2002, MNRAS, 330, 547

Trager, S. C., Worthey, G., Faber, S. M., Burstein, D. \& Gonzalez, J. J. 1998, ApJS, 116, 1

Trager, S. C., Faber, S. M., Worthey, G., \& Gonzalez, J. J. 2000, AJ, 119,1645 